\title{
VALOR DIAGNÓSTICO DE SEGUNDAS BIOPSIAS PROSTÁTICAS EN VARONES DE RIESGO. ESTUDIO ESTRATIFICADO POR VALOR DE PSA
}

\author{
J. CERVERA DEVAL, F.J. MORALES OLAYA, J. JORNET FAYOS, M. GONZÁLEZ AÑÓN
}

Fundación Instituto Valenciano de Oncología. Valencia.

Actas Urol Esp. 28 (9): 666-671, 2004

\section{RESUMEN}

VALOR DIAGNÓSTICO DE SEGUNDAS BIOPSIAS PROSTÁTICAS EN VARONES DE RIESGO. ESTUDIO ESTRATIFICADO POR VALOR DE PSA

OBJETIVO: Valorar el rendimiento diagnóstico de la segunda biopsia prostática (BP).

PACIENTES Y METODOS: Un total de 116 varones con BP previa de benignidad fueron sometidos a 2 o más BP por sextantes guiadas con ultrasonidos (US). Los criterios de inclusión fueron: BP previa sospechosa (PIN), PSA elevado, TR o US sospechoso.

RESULTADOS: El tiempo medio transcurrido entre la primera y siguiente biopsia fue de $13 \pm 11$ meses.

Se obtuvieron 35 malignas y 4 premalignas en las segundas biopsias realizadas, lo que da un rendimiento diagnóstico global del 33,6\%. Cuando estratificamos por valor de PSA, obtenemos que con PSA $<4 \mathrm{ng} / \mathrm{ml}$ el rendimiento fue de $23 \%$, para PSA $4-10 \mathrm{ng} / \mathrm{ml}$ el rendimiento fue de $32 \%$ y para PSA $>10 \mathrm{ng} / \mathrm{ml}$, de $34,6 \%$.

CONCLUSIÓN: La repetición de la biopsia seriada de próstata en pacientes de riesgo, mejora el rendimiento diagnóstico y elimina los falsos negativos de carcinoma. prostático.

PALABRAS CLAVE: Próstata. Biopsia prostática. Biopsia transrectal. Cáncer de próstata.

\section{ABSTRACT \\ DIAGNOSTIC VALUE OF THE SECOND PROSTATE BIOPSIES IN MALES OF RISK. STUDY STRATIFIED BY VALUE OF PSA}

OBJETIVE: To value the diagnostic yield of the second prostate biopsy (BP).

PATIENTS AND METHODS: To 116 males with BP previous to kindliness surrendered to 2 or more BP for sextants guided with ultrasounds (US). The criteria of inclusion were: BP previous suspicious (PIN), high PSA, TR or suspicious US.

RESULTS: The average time passed between the first and following biopsy was $13 \pm 11$ months.

35 malignant and 4 premalignant ones were obtained in the second realized biopsies, which gives a diagnostic global yield of $33.6 \%$. When we stratify for value of PSA, we obtain that with PSA $<4$ $\mathrm{ng} / \mathrm{ml}$ the yield was $23 \%$, for PSA $4-10 \mathrm{ng} / \mathrm{ml}$ the yield was $32 \%$ and for PSA $>10 \mathrm{ng} / \mathrm{ml}$ of 34,6\%.

CONCLUSION: The repetition of the serial biopsy of prostate in patients of risk, improves the diagnostic yield and eliminates the false negatives of prostate carcinoma. 
$\mathrm{E}^{\prime}$ manejo clínico y terapéutico del cáncer de próstata y el pronóstico de la enfermedad se encuentran íntimamente relacionados con la posibilidad de obtener un diagnóstico lo más precoz posible ${ }^{1}$

El uso del antígeno prostático específico (PSA) como test para seleccionar pacientes de riesgo ha contribuido notablemente en su diagnóstico precoz por su alta sensibilidad como indicador de alteraciones de la glándula prostática. Sin embargo su especificidad es menor de manera, que ante un aumento de los niveles de PSA, la pregunta que se plantea es si ese aumento es por cáncer o es debido a un proceso benigno. Varones con cifras de PSA superiores a 2,5 $\mathrm{ng} / \mathrm{ml}$ tienen una posibilidad de padecer cáncer en ese momento superior al $20 \%$ y si son mayores de $10 \mathrm{ng} / \mathrm{ml}$ es de un 50\%. Así mismo, varones con niveles de PSA $<2,5 \mathrm{ng} / \mathrm{ml}$ tienen una posibilidad del $1 \%$ de ser diagnosticados de enfermedad maligna de la próstata en los siguientes 4 años ${ }^{2}$.

La mayoría de los cánceres prostáticos son diagnosticados por biopsia transrectal (BTR). La BTR guiada por ultrasonidos es considerada la prueba de mayor valor para la detección de cáncer prostático ${ }^{3-5}$. Las indicaciones para este procedimiento incluyen fundamentalmente el tacto rectal (TR) sospechoso y el PSA elevado ${ }^{6}$. El TR posee un indudable valor aunque es un examen claramente subjetivo. Por otro lado, los niveles de PSA que se pueden considerar normales son variables dependiendo de la edad del paciente y del volumen de la próstata. La ecografía transrectal (ETR) no es un método capaz de descartar precozmente el cáncer de próstata si se usa de forma aislada, por su baja sensibilidad y especificidad. En la actualidad la ecografía es utilizada fundamentalmente para dirigir la aguja en la BTR. El uso de la ecografía tridimensional no ha alterado este papel. Con el Doppler color se mejora ligeramente la sensibilidad y el valor predictivo negativo de la ETR aunque no lo suficiente como para poder evitar la práctica de la BTR. El color puede ayudar a descubrir áreas sospechosas a las que dirigir la aguja de la biopsia ecoguiada $^{7,8}$. El uso de contrastes ecográficos basados en microburbujas proporciona información sobre la angiogénesis del tumor, ya que se trata de agentes intravasculares, pero no mejora significativamente la detección del cáncer de próstata $^{9}$. Recientes trabajos aprovechan el hecho de que las microburbujas pueden ser localmente destruidas mediante ondas sónicas liberando su contenido fuertemente ecogénico lo que aumentaría la señal en el tejido prostático normal, dejando hipoecogénicas las áreas tumorales $^{2}$. La resonancia magnética $(\mathrm{RM})$ con antena endorectal es la técnica de imagen adecuada para el estudio de extensión local y estadificación del cáncer de próstata, pero no para su diagnóstico precoz ${ }^{10}$. Sin embargo, utilizando la capacidad de la RM como técnica morfológica y funcional parece que su futuro es mucho más prometedor. La espectroscopia muestra depleción de citratos en el cáncer de próstata con respecto a otros metabolitos tales como colina y creatina. Codificando en color las áreas de relación anormal de metabolitos con la imagen anatómica de la RM se podría obtener un mapa tumoral ${ }^{2}$.

Pese a estos prometedores avances hasta el momento actual el antígeno específico prostático (PSA) es el parámetro más usado para la detección precoz del cáncer de próstata, como método de selección de pacientes candidatos a la BTR. Su uso generalizado ha provocado una disminución del número de pacientes con enfermedad metastásica en el momento del diagnóstico y una disminución de la tasa de mortalidad por cáncer prostático ${ }^{11}$. Los pacientes con cáncer de próstata tienen significativamente mayores niveles de PSA y mayor velocidad de PSA que aquellos en los que no se ha diagnosticado cáncer. Sin embargo, los programas de cribado poblacional basados en el PSA han mostrado que, aproximadamente, un 9\% de varones asintomáticos muestran valores elevados de PSA, pero solo la tercera parte de ellos tienen cáncer detectado en una primera evaluación ${ }^{12,13}$. Los dos tercios restantes con diagnóstico negativo de cáncer tienen cifras elevadas de PSA por hiperplasia benigna, prostatitis o incluso por cáncer no detectado. Para descartar esta última posibilidad se plantea frecuentemente la necesidad de realizar una segunda BTR.

Nuestro objetivo en el presente trabajo fue valorar el rendimiento diagnóstico de las segundas BTR de la próstata según el valor de PSA. 


\section{MATERIAL Y MÉTODOS}

De un total de 853 varones con una primera BTR con diagnóstico negativo para malignidad, 116 pacientes se sometieron a 2 o más biopsias por sextantes guiadas con ultrasonidos. Los criterios de inclusión fueron una BTR previa sospechosa (PINneoplasia intraepitelial prostática), un PSA elevado o en aumento progresivo, un TR sugerente y un estudio ultrasonográfico transrectal sospechoso.

Realizamos un TR previo al procedimiento biópsico, categorizándolo en sospechoso o no. Consideramos un TR positivo cuando hubo anormalidades sugestivas de cáncer.

El volumen prostático fue calculado con ultrasonidos, obteniéndolo de la multiplicación de los tres diámetros máximos medidos en dos planos por 0,526. No se consideró el volumen prostático para aumentar el número de muestras por procedimiento biópsico realizado.

Para la biopsia por sextantes utilizamos aguja de 18G (Acecut-TSK) con disparo y corte automático, la cual permite obtener cilindros de hasta 20 $\mathrm{mm}$ de longitud. Se tomaron muestras del ápex, de la porción medio-lateral y de la base de cada uno de los lados de la glándula prostática. Además en los casos en los que hubo hallazgos ecográficos sugestivos de neoformación, se procedió a completar con muestras adicionales de dichas alteraciones.

$\mathrm{Al}$ igual que otros autores ${ }^{14,15}$ agrupamos con los benignos los PIN grado I y II, y como premalignos a los PIN grado III.

\section{RESULTADOS}

La media de edad de los varones incluidos en este estudio fue de $69 \pm 6$ años (máxima de 84 y mínima de 53 años) con volumen prostático medio de 65,4 $\pm 31,3 \mathrm{cc}$ (rango 20-140 cc). El tiempo medio transcurrido entre la primera y siguiente biopsia fue de $13 \pm 11$ meses (rango 162 meses y moda y mediana de 6 meses).

La indicación de segunda biopsia fue por TR sospechoso en 23 pacientes, elevación de PSA en 50 pacientes, TR sospechoso con elevación de PSA en 26 pacientes, por biopsia previa (PIN) en 8 pacientes (5 premalignos), y por estudio ultrasonográfico transrectal sospechoso en 9 pacientes. El resultado histológico de la segunda biopsia según la indicación queda reflejado en la Tabla 1.
Tabla 1

Resultado histopatológico según la indicación de las segundas biopsias

\begin{tabular}{lcccc}
\hline & Benigna & Maligna & Premaligna & Total \\
\hline $\begin{array}{l}\text { TR } \\
\text { sospechoso }\end{array}$ & 16 & 6 & 1 & 23 \\
$\begin{array}{l}\text { Elevación } \\
\text { PSA }\end{array}$ & 38 & 10 & 2 & 50 \\
TR y PSA & 9 & 17 & & 26 \\
$\begin{array}{l}\text { Por biopsia } \\
\text { previa (PIN) }\end{array}$ & 6 & 1 & 1 & 8 \\
$\begin{array}{l}\text { Por US } \\
\text { previo } \\
\text { sospechoso }\end{array}$ & 8 & 1 & & 9 \\
Total & 77 & 35 & 4 & 116 \\
\hline
\end{tabular}

US: Ultrasonografia transrectal

Entre los 116 varones que se sometieron a dos o más biopsias prostáticas se obtuvieron 35 cánceres y 4 lesiones premalignas, lo que da un rendimiento diagnóstico global de 33,6\% (Tabla 2).

Tabla II

Resultado de la segunda biopsia

\begin{tabular}{llcccc}
\hline & & \multicolumn{3}{c}{$\begin{array}{c}\text { Anatomia patológica } \\
\text { definitiva }\end{array}$} & \\
\cline { 3 - 5 } & & Benigna & Maligna & Premaligna & Total \\
\hline $\begin{array}{l}\text { Anat. pat. } \\
\text { inicial }\end{array}$ & Benigna & 74 & 34 & 3 & 111 \\
& Premaligna & 3 & 1 & 1 & 5 \\
& Total & 77 & 35 & 4 & 116 \\
\hline
\end{tabular}

Estratificando por valor de PSA obtuvimos los siguientes resultados: con $\mathrm{PSA}<4 \mathrm{ng} / \mathrm{ml}$ hubo 13 casos de indicación de segunda biopsia en los que se encontraron 3 cánceres, lo que da un rendimiento diagnóstico de $23 \%$. Con PSA entre 4-10 $\mathrm{ng} / \mathrm{ml}$ hubo 28 casos de indicación biópsica, de los que 2 resultaron PIN de alto grado y 7 fueron cáncer, de modo que el rendimiento diagnóstico fue del $32 \%$. Finalmente, para PSA $>10 \mathrm{ng} / \mathrm{ml}$ se estudiaron 75 casos, encontrando 2 PIN de alto grado y 24 carcinomas, por lo que el rendimiento fue del $34,6 \%$.

\section{DISCUSIÓN}

En la revisión de McNeal et al. encontraron que la distribución de cáncer prostático se localizó un 
$68 \%$ en la zona periférica, el $8 \%$ en la porción central y el $24 \%$ en las zonas de transición ${ }^{16}$, elevándose al $53 \%$ en la zona de transición en otra serie $^{17}$. La recomendación de que a todos los pacientes con TR negativo y PSA elevado se realice biopsias por sextantes incluyendo tanto la zona periférica como la zona de transición parece lógica dada la distribución anatómica del cáncer de próstata ${ }^{18}$. Hay estudios que han revelado que los cánceres de la zona de transición son similares en tamaño a los cánceres de las zonas periféricas, pero que habitualmente tienen menor grado de Gleason, menor invasión capsular y de vesículas seminales y una mínima asociación con PIN de alto grado ${ }^{19,20}$.

En nuestra serie, al igual que en la Keetch y Catalona no encontramos diferencias estadísticamente significativas en la detección de cáncer entre los pacientes que se realizaron una segunda biopsia y los que se realizaron tres o más biopsias. Estos autores biopsiando la zona periférica encontraron un 19\% de cánceres en una segunda biopsia y un $8 \%$ en una tercera biopsia.

Nosotros tampoco encontramos diferencias significativas en la media de edad, en la sospecha del TR, ni en la concentración de PSA. Sin embargo, los pacientes con cáncer también tenían un menor volumen prostático y un mayor PSA que los que no lo tenían ${ }^{21}$.

En nuestra serie de 116 varones incluidos, hemos realizado una segunda biopsia en el $84,5 \%$ de los casos, hasta una tercera biopsia en el $11,2 \%$, y cuatro o más biopsias en el $4,3 \%$ de los casos. La indicación más frecuente para la repetición biópsica fue la elevación de PSA (36\%), por TR sospechoso se repitieron el $19,8 \%$ y por elevación de PSA con TR sospechoso en el 22,4\% de los casos. Otras indicaciones fueron por PIN en biopsia previa $(6,9 \%)$ y por algún hallazgo ultrasonográfico $(7,8 \%)$.

Los resultados de Fleshner et al. ${ }^{22}$ demuestran que de las variables analizadas solo el PSA y una ETR anormal (lesión de 0,1 cc o mayor) fueron factores de riesgo significativos para repetir la biopsia TR. Por el contrario la edad media, la densidad del PSA y el intervalo de la biopsia no presentaron diferencias estadísticamente significativas en varones con biopsia TR repetida positiva o negativa. Además el TR anormal, densidad de PSA $>0,15 \mathrm{ng} / \mathrm{ml} / \mathrm{cc}$, la velocidad de PSA $>0,75 \mathrm{ml} /$ año, la historia familiar y los PIN grado II y III o atipia tampoco presentaron asociación con cáncer en las repeticiones de biopsia. En este mismo articulo los autores demuestran con su análisis multivariante que únicamente un PSA $>20 \mathrm{ng} / \mathrm{ml}$ y una ecografía TR anormal fueron predictivas para una biopsia TR positiva, aunque solo el PSA $>20 \mathrm{ng} / \mathrm{ml}$ fue significativo en esta multivariante. Sin embargo hay referencias de que con un PSA $>10 \mathrm{ng} / \mathrm{ml}$ se encontraron un $41 \%$ de segundas biopsias positivas ${ }^{23}$.

En una serie sobre 427 varones con PSA persistentemente elevado, sólo el 19\% de ellos tuvieron una segunda biopsia positiva. En esta publicación el PSA y la velocidad de PSA fueron factores de riesgo significativos para las biopsias positivas $^{24}$. En otra serie, el 20\% de varones con una BTR guiada con ultrasonidos negativa tuvieron una segunda biopsia positiva, aunque en la misma, no determinaron los factores de riesgo ${ }^{14}$.

Sin embargo, muchas series han demostrado que cuando se sospecha cáncer prostático, debido a un PSA elevado, o por un TR o estudio ecográfico sospechoso, entre un 19 y un $28 \%$ de casos son diagnosticados cuando se realizan las segundas biopsias por sextantes ${ }^{25}$. Este porcentaje puede aumentar hasta un $3 \%$ más si se obtienen 6 o más muestras ${ }^{26}$. Otras series encuentran cáncer en sólo un 10\% de segundas biopsias cuando la previa fue negativa ${ }^{27}$, esta diferencia los autores la atribuyen a la técnica biópsica o al hecho de que la biopsia se repitió con sólo 6 semanas de intervalo.

Ukimura et al. estudiaron 193 varones con biopsia inicial negativa de los que 51 (26\%) detectaron cáncer en la siguiente biopsia, concluyendo que en su serie entre PSA total, densidad de PSA, PSA referido a la edad, velocidad de PSA y PSA referido al volumen, los únicos parámetros estadísticamente significativos fueron el PSA total y el PSA referido al volumen ${ }^{28}$.

Djavan et al. ${ }^{27}$ refieren que de 1.051 varones con valores de PSA de 4 a $10 \mathrm{ng} / \mathrm{ml}$, encontraron 231 casos $(22 \%)$ de cáncer prostático en la primera biopsia y solo 83 casos de los 820 restantes en la segunda biopsia realizada 6 semanas más tarde, teniendo estos últimos un volumen prostático significativamente mayor, de modo que se 
podría justificar la menor detección de cáncer en las segundas biopsias por el mayor volumen prostático y por consiguiente de tejido hiperplásico en los casos de hiperplasia benigna de próstata con PSA elevado ${ }^{29,30}$. En la serie de Levine et al. ${ }^{31}$ los cánceres prostáticos fueron detectados en el $43 \%$ de próstatas con volumen inferior a 30 cc, en el $27 \%$ con volúmenes de 30 a 50 cc y en el $24 \%$ con volúmenes superiores a 50 cc. En la serie de Uzzo et al. ${ }^{30}$ encuentran un 23\% de cánceres en próstatas de igual o más de 50 cc de volumen comparadas con un $38 \%$ de próstatas con volúmenes inferiores $(\mathrm{p}<0,01)$ concluyendo que el volumen juega un papel importante en la detección biópsica sobre todo en las próstatas voluminosas. Respecto al PSA, obtienen histopatología de adenocarcinoma en el 20\% de varones con PSA normal. Nosotros con valores de PSA <4 $\mathrm{ng} / \mathrm{ml}$ encontramos 3 casos de adenocarcinomas de un total de 13 varones biopsiados, mientras que con valores de PSA de 4-10 ng/ml obtuvimos 9 casos y con valores superiores a $10 \mathrm{ng} / \mathrm{ml}, 26$ casos.

Hay varias explicaciones para el bajo número de biopsias positivas (siempre referido al PSA). La primera es que la biopsia por sextantes proporciona pequeñas muestras de tejido prostático. Cada cilindro pesa aproximadamente 0,002 gr (Reuter V. Comunicación personal) por lo tanto las muestras de las biopsias por sextantes son aproximadamente el 0,02\% del total de la próstata (peso medio calculado por ETR en esta serie de 62,5 gr). Otra explicación es el número de pacientes con cáncer no palpable (T1c), ya que los pequeños tumores son más difíciles de descubrir. La naturaleza multifocal y la alta prevalencia del cáncer de próstata hacen que la detección sea por casualidad. Otra posible explicación es que la incidencia actual de cáncer de próstata es independiente del volumen y las bajas tasas de detección sean debidas a un inadecuado muestreo de la glándula, por lo que aumentando el número de biopsias disminuye el margen de error. No obstante se acepta que la incidencia de cáncer prostático en una segunda biopsia varía del 19 al 25\% ${ }^{24}$. Así, se ha diseñado un modelo teórico basado en la European Prostatic Cancer Detection (EPCD) en el que se toma la edad del paciente y el volumen de la próstata para definir el número de pases a realizar con el fin de obtener un rendimiento del 90\% en la detección de cáncer prostático. El número de tomas recomendado oscila entre 8 para volúmenes prostáticos inferiores a 29 cc en pacientes de menos de 50 años y 14 en próstatas de más de 70 cc de volumen y pacientes de más de 70 años. Para la indicación de segunda biopsia Djavan et al. proponen un algoritmo basado en el PSA, relación de PSA libre con respecto al total y valoración de PSA en la zona de transición medido por histoquimia de las muestras obtenidas. Para estos autores una biopsia prostática negativa con PSA $>10 \mathrm{ng} / \mathrm{ml}$ es siempre indicación de rebiopsia ${ }^{32}$.

\section{CONCLUSIÓN}

La repetición de la biopsia seriada de próstata en pacientes con sospecha clínica, analitica o ecográfica y un resultado anatomopatológico de benignidad en la primera biopsia, mejora el rendimiento diagnóstico global de cáncer prostático en un 33,6\%. Los principales factores de riesgo son un PSA de 4-10 ng/ml (32\%) y un PSA > 10 $\mathrm{ng} / \mathrm{ml}(34,6 \%)$.

\section{REFERENCIAS}

1. Rifkin MD. Personal presentation. Chicago. RSNA Categorical Course of Genitourinary Radiology 1994:175-182.

2. Thornbury JR, Ornstein DK, Choyke PL, Curtis P. Langlotz and Jeffrey C. Weinreb prostate cancer. What is the future role for imaging? AJR 2001;176: 17-22.

3. Hodge KK, McNeal JE, Terris MK, Stamey TA. Random systematic versus directed ultrasound guided transrectal core biopsies of the prostate. J Urol 1989 jul;142 (1):71-74, discussion 74-75.

4. Garber SJ, Goldenberg SL, Cooperberg PL, Wong AD, Bilby JH, Mathieson JR. Systematic transrectal ultrasound-guided biopsy of the prostate. Canad Ass Rad 1994;45:387-390.

5. Rabbani F, Stroumbakis N, Kava BR, Cookson MS, Fair WR. Incidence and clinical significance of false-negative sextant prostate biopsies. J Urol 1998 apr; 159(4):1247-1250.

6. Stamey TA, McNeal JE. Adenocarcinoma of the prostate. En: Campbell's Urology. 6th ed. Edited by Walsh PC, Retik AB, Stamey TA and Vaughan ED Jr. Philadelphia, W.B. Saunders Co. 1992;2(29):1159-1221.

7. Morales FJ, Dualde D, Martínez F, Labrador T, Vidal P, Gordo G. Valor de la ecografía transrectal ecodirigida en la detección precoz del cáncer de próstata. Resultados de un estudio sobre 700 casos. Radiología 1999;41(5):343350 .

8. Morales FJ, Jornet J, Cervera J, Labrador T. Utilidad de la ecografia transrectal con Doppler color en el diagnóstico del cáncer de próstata. Estudio de 101 casos. Radiología 2001; 43(6):279-285. 
9. Jornet J, Morales J, Cervera J, Dualde D, Marhuenda A. Utilidad de la ecografía transrectal con potenciador de señal ecográfico frente al Doppler-color basal en el diagnóstico del cáncer de próstata. Comunicación personal. Congreso Nacional SERAM. 2002. Las Palmas de G.C.

10. Rorvik J, Halvorsen OJ, Albrektsen G, Ersland L, Daehlin L, Haukaas S. MRI with an endorectal coil for staging of clinically localised prostate cancer prior to radical prostatectomy. European Radiology 1999;9(1):29-34.

11. Greenlee RT, Murray T, Boldens S, Wingo PA. Cancer statistics 2000. CA Cancer J Clin 2000;50(1): 7-33.

12. Catalona WJ, Smith DS, Ratliff TL, Dodds KM, Coplen DE, Yuan JJ, Petros JA, Andriole GL. Measurement of prostate-specific antigen in serum ass a screening test for prostate cancer. New Engl J Med 1991 apr 25;324(17): 1156-1161.

13. Brawer MK, Chetner MP, Beatie J, Buchner DM, Vessella RL, Lange PH. Screening for prostatic carcinoma with prostate specific antigen. J Urol 1992;2(147):841-845.

14. Ellis WJ, Brawer MK. Repeat prostate needle biopsy: who needs). J Urol 1995 may;153:1496-1498.

15. Bostwick DG, Qian J, Frankel K. The incidence of high grade prostatic intraepithelial neoplasia in needle biopsies. J Urol 1995 nov;154(5):1791-1794.

16. McNeal JE, Redwine EA, Freiha FS, Stamey TA. Zonal distribution of prostatic adenocarcinoma: correlation with histologic pattern and direction of spread. Amer J Surg Path 1988 dec;12(12):897-906.

17. Lui PD, Terris MK, McNeal JE, Stamey TA. Indications for ultrasound guided transition zone biopsies in the detection of prostate cancer. J Urol 1995 mar;153(3Pt 2):1000-1003.

18. Cupp MR, Oesterling JE. Detecting early prostate cancer. AUA update series 1993;12:258-263.

19. Greene DR, Wheeler TM, Egawa S, Dunn JK, Scardino PT. A comparison of the morphological features of cancer arising in the transition zone and in the peripheral zone of the prostate. J Urol 1991 oct;146(4):1069-1076.

20. Lee F, Siders DB, Torp-Pederson ST, Kiercht JL, McHugh TA, Mitchelle AE. Prostate cancer: transrectal ultrasound and pathology comparison. A preliminary study of outer gland (peripheral and central zones) and inner gland (transition zone). Cancer 1991 feb 15;67(4 suppl):11321142.

21. Keetch DW, Catalona WJ. Prostatic transition zone biopsies in men with previous negative biopsies and persistently elevated serum prostate specific antigen values. J Urol 1995 nov;154(5):1795-1797.
22. Fleshner NE, O`Sullivan M, Fair WR. Prevalence an predictors of a repeat transrectal ultrasound guided needle biopsy of the prostate. J Urol 1997 augt; 158(2):505-508.

23. Catalona WJ, Smith DS, Ratliff TL, Busler JW. Detection of organ-confined prostate cancer is increase through prostate-specific antigen-based screening. JAMA 1993 augt 25;270(8):948-954.

24. Keetch DW, Catalona WJ, Smith DS. Serial prostatic biopsies in men with persistently elevated serum prostate specific antigen values. J Urol 1994 jun; 151(6):1571-1574.

25. Roehrborn CG, Pickens GJ, Sanders JS. Diagnostic yield of repeated transrectal ultrasound-guided biopsies stratified by specific histopathologic diagnoses and prostate specific antigen levels. Urology 1996 mar;47(3):347-352.

26. Eskew AL, Bare RL, McCullough DL. Systematic 5 regions prostate biopsy is superior to sextant method for diagnosing carcinoma of the prostate. J Urol 1997 jan;157(1):199202; discussion 202-203.

27. Djavan B, Zlotta A, Renmzi M, Ghawidel K, Basharkhah A, Schulman C, Marberger H. Optimal predictors of prostate cancer on repeat prostate biopsy: a prospective study of 1051 men. J Urol 2000 apr;163(4):1144-1148; discussion 1148-1149.

28. Ukimura O, Durrani O, Babaian RJ. Role of PSA and its indices in determining the need for repeat prostate biopsies. Urology 1997 jul;50(1):66-72.

29. Karakiewicz PI, Bazinet M, Aprikian AG, Trudel C, Aronson S, Nachabe M, Peloquini F, Dessureault J, Goyal MS, Beguin LR, Elihilali MM. Outcome of sextant biopsy according to gland volume. Urology 1997 jan;49(1):55-59.

30. Uzzo RG, Wei JT, Waldbaum RS, Perlmutter ASP, Byrne JC, Vaughan ED Jr. The influence of prostatic size on cancer detection. Urology 1995 dec; 46(6):831-836.

31. Levine MA, Ittman M, Melamed J, Lepor H. Two consecutive sets of transrectal ultrasound guided sextant biopsies of the prostate for the detection of prostate cancer. J Urol 1998 feb;159(2):471-475.

32. Djavan B, Remzi M, Schulman CC, Marberger M, Zlotta AR. Repeat prostate biopsy: who, how and when? European Urology 2002;42:93-103.

Dr. J. Cervera Deval

Fundación Instituto Valenciano de Oncología

C/ Prof. Beltrán Báguena, 8

46009 Valencia

(Trabajo recibido el 18 junio de 2004) 